\title{
Effective wavelength as a universal parameter of hyperspectral light radiance upwelling from the sea
}

\author{
Vladimir I. Haltrin, ${ }^{\dagger}$ Robert A. Arnone,${ }^{\dagger}$ and Vyacheslav A. Urdenko ${ }^{\circ}$ \\ $\dagger$ Naval Research Laboratory, Ocean Optics Section, Code 7333, \\ Stennis Space Center, MS 39529-5004, USA* \\ 'Marine Hydrophysical Institute, Ukrainian Academy of Sciences, \\ 2a Kapitanskaya St., Sevastopol, 99000, Crimea, Ukraine
}

\begin{abstract}
This paper presents experimentally obtained relationships between effective wavelength and a number of important seawater parameters such as dominant wavelength, different color indices, downward diffuse attenuation coefficient, absorption coefficient, seawater effective optical thickness, and a chlorophyll content. A number of regression relationships that connect hyperspectral measurements with listed bio-optical properties are proposed and discussed.
\end{abstract}

Keywords: marine optics, scattering, sea water, optical properties

\section{INTRODUCTION}

Processing of optical satellite data corrected from atmospheric effects is usually consists in correlating different combinations of upwelling radiances with optical and biological parameters of seawater. When the number of channels is small it is possible to correlate almost all combinations of radiances with bio-optical properties. In processing hyperspectral data the number of channels is so large that it is impossible to effectively utilize all hyperspectral information using only radiances at certain wavelengths. In order to effectively process all hyperspectral information we need to introduce a new set of integral parameters that are related to all spectral channels. One of the simplest parameter is a normalized first moment of visible spectrum in relation to wavelength. We name this parameter as an effective wavelength of upwelling hyperspectral radiance.

This paper discusses different experimentally obtained relationships between effective wavelength and a number of important seawater parameters such as CIE dominant wavelength, different color indices, downward diffuse attenuation coefficient, absorption coefficient, seawater effective optical thickness, and a chlorophyll concentration. A number of regression relationships that connect hyperspectral measurements with listed bio-optical properties are proposed and discussed. It was shown that an effective wavelength is a universal parameter that connects hyperspectral optical measurements with a number of optical properties and effective chlorophyll concentration.

* Further author information: e-mail: haltrin@nrlssc.navy.mil; Telephone: 228-688-4528; Fax: 228-688-4149.

Ocean Optics:Remote Sensing and Underwater Imaging, Gary D. Gilbert, Robert J. Frouin, Editors, Proceedings of SPIE Vol. 4488 (2002) @ 2002 SPIE • 0277-786X/02/\$15.00 


\section{INTRODUCTION}

As an integral parameter of multispectral quantity $\Phi(\lambda)$, such as upwelling radiance $L(\lambda)$, irradiance $E(\lambda)$, remote sensing reflection coefficient $r(\lambda)$, or diffuse reflection coefficient $R(\lambda)$, etc. let us choose the following value,

$$
\lambda_{\text {eff }}(\Phi)=\int_{\lambda_{1}}^{\lambda_{2}} \lambda \Phi(\lambda) d \lambda / \int_{\lambda_{1}}^{\lambda_{2}} \Phi(\lambda) d \lambda
$$

It is convenient to name this parameter as an effective wavelength over spectrum $\Phi(\lambda)$ in the range of $\left\{\lambda_{1}, \lambda_{2}\right\}$. If $\Phi(\lambda)=E_{\uparrow}(\lambda), \lambda_{\text {eff }}$ has a physical meaning: it is reversely proportional to the average energy of quanta in the range of $\lambda_{1} \leq \lambda \leq \lambda_{2}$, i.e. $\lambda_{\text {eff }}\left(E_{\uparrow}\right)=h c Q / W$, where

$$
Q=\frac{1}{h c} \int_{\lambda_{1}}^{\lambda_{2}} \lambda E_{\uparrow}(\lambda) d \lambda
$$

is quantum irradiance from below, and

$$
W=\int_{\lambda_{1}}^{\lambda_{2}} E_{\uparrow}(\lambda) d \lambda
$$

is total energetic irradiance, $h$ is the Plank's constant, $c$ is the speed of light in vacuum. In the case of $\lambda_{1}=350 \mathrm{~nm}, \lambda_{2}=700 \mathrm{~nm}$, that we will use in this paper, and $\Phi(\lambda)=E_{\uparrow}(\lambda)$ Eq. (1) determines the effective wavelength of photo-synthetically active radiation. Because spectral shapes of $E(\lambda)$ and $L(\lambda)$ are identical [1], $\lambda_{\text {eff }}\left(E_{\uparrow}\right) \cong \lambda_{\text {eff }}\left(L_{\uparrow}\right)$. Numerical estimates show that normalizing $E(\lambda)$ and $L(\lambda)$ by downward irradiance $E_{\downarrow}(\lambda)$, which corresponds to $\Phi(\lambda)=R(\lambda)$ and $\Phi(\lambda)=r(\lambda)$, changes values of $\lambda_{\text {eff }}$ less than $0.5 \%$. Analysis of data collected during our joint shipborn and airborn experiments shows that effective wavelength weakly varies with the change in lightning conditions, and optical properties of atmosphere. At the same time $\lambda_{\text {eff }}$ is strongly coupled with inherent optical properties of the water mass, light field parameters of the water, and concentrations of dissolved and suspended matter [2].

\section{CONNECTION BETWEEN EFFECTIVE WAVELENGTH OF UPWELLING RADIATION AND LIGHT FIELD PARAMETERS OF SEAWATER}

Being an integral property of the light upwelling from the seawater, specifically, being a wavelength averaged over light spectrum upwelling from the sea, the effective wavelength $\lambda_{\text {eff }}$ is particularly sensitive to the highly energetic components of sea light that determine the color of the water mass. Comparison of computed values of effective $\lambda_{\text {eff }}$ and dominant $\lambda_{D}[3,4]$ wavelengths revealed strong coupling between these properties. This coupling may be expressed by the following regressions:

$$
\begin{gathered}
\lambda_{D}=213.6+0.56 \lambda_{\text {eff }}, \quad 459<\lambda_{\text {eff }}<497 \mathrm{~nm}, \quad(N=24, r=0.96), \\
\lambda_{D}=-1038.4+3.07 \lambda_{\text {eff }}, \quad 498<\lambda_{\text {eff }}<521 \mathrm{~nm}, \quad(N=25, r=0.93) .
\end{gathered}
$$

The color index, which was widely used in hydro-optical measurements since its introduction by Jerlov [5], also correlates nicely with the effective wavelength: 


$$
\begin{aligned}
& \lg J\left(\lambda_{n}, \lambda_{k}\right)=-A_{1}+B_{1} \lambda_{\text {eff }}, \\
& \lg \lambda_{\text {eff }}=A_{2}+B_{2} J\left(\lambda_{n}, \lambda_{k}\right),
\end{aligned}
$$

here and everywhere in this paper $\lg$ means a logarithm of base 10 .

Equations (4) and (5) are given for different combinations of wavelength pairs $\left\{\lambda_{n}, \lambda_{k}\right\}$, and coefficients $A_{1}$, $B_{1}, A_{2}$, and $B_{2}$ in Eqs. (4)-(5) are given in Tables $1 \mathrm{a}, 1 \mathrm{~b}, 2 \mathrm{a}$, and $2 \mathrm{~b}$, respectively.

Not all color indices $J\left(\lambda_{n}, \lambda_{k}\right)=L_{\uparrow}\left(\lambda_{n}\right) / L_{\uparrow}\left(\lambda_{k}\right)$ correlate with $\lambda_{\text {eff }}$ equally well, but in all cases presented in Tables 1 and 2 the correlation coefficients are larger than 0.8 . For majority of the pairs presented here the correlation coefficient exceeds 0.9 and reaches maximum of $r=0.996$ for $J(580,420)$ [6].

In spite of the fact that the choice of wavelengths $\lambda_{n}$ and $\lambda_{k}$ is usually related to the maximums of phytoplankton absorption, there is no general agreement concerning the values of these wavelengths. This causes difficulties in comparing results of color index measurements obtained by different authors. The joint use of Eqs. (6) and (7) allow us to compare and generalize such previously incomparable measurements.

Some optical classifications of water masses [7-10] use spectral distribution of downward diffuse attenuation coefficient $k_{\downarrow}(\lambda)$ as their basis. This is possible because the $k_{\downarrow}(\lambda)$ averaged over thick subsurface layer of water

Table 1a. Numerical values of coefficient $A_{1}$ in Eq. (6).

\begin{tabular}{|c|c|c|c|c|c|c|c|}
\hline$\lambda_{n} \backslash \lambda_{k}$ & 410 & 420 & 430 & 440 & 450 & 460 & 470 \\
\hline 470 & 3.25 & 2.94 & 2.65 & 2.15 & 1.18 & 0.5 & - \\
480 & 4.06 & 3.75 & 3.47 & 2.95 & 1.97 & 1.39 & 0.78 \\
490 & 4.66 & 4.36 & 4.05 & 3.56 & 2.57 & 1.98 & 1.4 \\
500 & 6.12 & 5.81 & 5.51 & 5.00 & 4.02 & 3.43 & 2.84 \\
510 & 7.68 & 7.4 & 7.09 & 6.59 & 5.6 & 5.02 & 4.45 \\
520 & 9.65 & 9.29 & 8.98 & 8.49 & 7.52 & 6.95 & 6.34 \\
530 & 10.30 & 10.18 & 9.71 & 9.41 & 8.38 & 7.76 & 7.21 \\
540 & 11.17 & 10.73 & 10.55 & 10.05 & 9.95 & 8.49 & 7.89 \\
550 & 12.25 & 11.89 & 11.63 & 11.20 & 10.18 & 9.81 & 9.05 \\
560 & 13.32 & 12.98 & 12.71 & 12.15 & 11.21 & 10.21 & 10.02 \\
570 & 13.43 & 13.19 & 12.94 & 12.45 & 11.47 & 10.87 & 10.32 \\
580 & 14.13 & 13.88 & 13.61 & 13.09 & 12.12 & 11.50 & 10.92 \\
590 & 13.35 & 13.01 & 12.69 & 12.21 & 11.31 & 10.60 & 9.59 \\
\hline
\end{tabular}

Table $1 \mathrm{~b}$. Numerical values of coefficient $B_{1} \cdot 10^{3}$ in Eq. (6).

\begin{tabular}{|c|c|c|c|c|c|c|c|}
\hline$\lambda_{n} \backslash \lambda_{k}$ & 410 & 420 & 430 & 440 & 450 & 460 & 470 \\
\hline 470 & 6.7 & 6.1 & 5.5 & 4.5 & 2.5 & 1.2 & - \\
480 & 8.4 & 7.8 & 7.2 & 6.2 & 4.2 & 2.9 & 1.6 \\
490 & 9.6 & 9.0 & 8.4 & 7.4 & 5.4 & 4.1 & 2.9 \\
500 & 13.0 & 12.0 & 11.0 & 10.0 & 8.3 & 7.0 & 5.8 \\
510 & 16.0 & 15 & 14 & 13 & 11 & 10 & 9.0 \\
520 & 20.0 & 19 & 18 & 17 & 15 & 14 & 13 \\
530 & 21.0 & 20.6 & 19.7 & 19.1 & 16.9 & 15.6 & 14.5 \\
540 & 22.6 & 21.7 & 21.4 & 20.3 & 18.3 & 17.1 & 15.8 \\
550 & 24.7 & 24.0 & 23.5 & 22.6 & 20.5 & 19.7 & 18.1 \\
560 & 26.8 & 28.2 & 25.6 & 24.5 & 22.5 & 21.3 & 20.0 \\
570 & 27.0 & 26.6 & 26.1 & 25.0 & 23.0 & 21.8 & 20.6 \\
580 & 28.1 & 27.7 & 27.2 & 26.1 & 24.1 & 22.6 & 21.5 \\
590 & 26.3 & 25.7 & 25.1 & 24.1 & 22.2 & 20.7 & 18.6 \\
\hline
\end{tabular}


under combined illumination of the sun and sky (with solar zenith angles higher than $45^{\circ}$ ) depends only on inherent optical properties of seawater. We obtained the following equation that connects spectral downward diffuse attenuation coefficient $k_{\downarrow}(\lambda)$ with the effective wavelength computed using upwelling radiation spectra in its visible part:

$$
\lg k_{\downarrow}(\lambda)=A_{3}(\lambda)+B_{3}(\lambda) \lambda_{\text {eff }}, \ldots 459<\lambda_{\text {eff }}<521 \mathrm{~nm}, \quad(N=49, r-\text { see Tab. } 3),
$$

here $k_{\downarrow}(\lambda)$ is downward diffuse attenuation coefficient averaged over upper sea layer in $\mathrm{m}^{-1}$. Coefficients $A_{3}$ and $B_{3}$ are given in Table 3. In all considered range of wavelengths, from 410 to $580 \mathrm{~nm}$, correlation coefficients are very high with maximum value of $r=0.98$ at $\lambda=430 \div 440 \mathrm{~nm}$. Comparison of additional measurements, not included in derivation of Eq. (8), showed a satisfactory agreement with the prediction given by Eq. (8).

Integral downward diffuse attenuation coefficient is connected with $z_{Q}(1 \%)$, the depth at which quantum irradiance $Q$, given by Eq. (2), is attenuated 100 times, by the following equation:

Table 2a. Numerical values of coefficient $A_{2}$ in Eq. (7).

\begin{tabular}{|c|c|c|c|c|c|c|c|}
\hline$\lambda_{n} \backslash \lambda_{k}$ & 410 & 420 & 430 & 440 & 450 & 460 & 470 \\
\hline 470 & 488.2 & 484.6 & 483.3 & 482.7 & 481.7 & 481.8 & - \\
480 & 486.7 & 483.3 & 482.1 & 481.1 & 479.5 & 478.3 & 482.3 \\
490 & 487.0 & 484.3 & 483.3 & 483.2 & 483.1 & 483.7 & 486.4 \\
500 & 488.8 & 486.3 & 485.8 & 486.1 & 486.5 & 487.4 & 490.0 \\
510 & 491.7 & 498.8 & 489.6 & 490.3 & 491.4 & 492.7 & 495.1 \\
520 & 494.0 & 492.5 & 492.4 & 493.2 & 494.4 & 495.6 & 497.7 \\
530 & 494.6 & 493.1 & 493.2 & 493.8 & 495.0 & 496.1 & 497.9 \\
540 & 494.9 & 493.8 & 493.5 & 494.2 & 495.4 & 496.4 & 498.1 \\
550 & 496.1 & 495.0 & 495.0 & 495.6 & 486.7 & 497.3 & 499.2 \\
560 & 496.7 & 495.7 & 495.7 & 496.2 & 497.4 & 498.4 & 499.6 \\
570 & 497.7 & 496.4 & 496.4 & 497.2 & 498.3 & 499.2 & 500.5 \\
580 & 501.9 & 500.9 & 501.0 & 501.8 & 503.2 & 504.3 & 505.8 \\
590 & 506.2 & 505.1 & 505.2 & 506.4 & 508.0 & 509.5 & 510.0 \\
\hline
\end{tabular}

Table 2b. Numerical values of coefficient $B_{2}$ in Eq. (7).

\begin{tabular}{|c|c|c|c|c|c|c|c|}
\hline$\lambda_{n} \backslash \lambda_{k}$ & 410 & 420 & 430 & 440 & 450 & 460 & 470 \\
\hline 470 & 83.3 & 98.9 & 115.3 & 153.7 & 238.7 & 352.7 & - \\
480 & 80.0 & 90.7 & 102.7 & 131.9 & 198.4 & 286.9 & 372.7 \\
490 & 68.1 & 74.9 & 84.0 & 98.1 & 121.4 & 139.5 & 139.2 \\
500 & 65.1 & 68.5 & 74.5 & 85.7 & 107.2 & 126.9 & 148.4 \\
510 & 56.3 & 59.2 & 62.9 & 69.6 & 82.9 & 98.2 & 103.4 \\
520 & 47.3 & 49.6 & 51.9 & 55.9 & 63.7 & 69.2 & 75.5 \\
530 & 44.5 & 46.3 & 47.5 & 51.0 & 57.7 & 62.3 & 66.7 \\
540 & 42.5 & 44.5 & 45.7 & 48.2 & 53.6 & 57.1 & 61.0 \\
550 & 39.4 & 40.7 & 41.8 & 43.6 & 48.0 & 49.5 & 53.4 \\
560 & 36.6 & 37.7 & 38.5 & 40.3 & 43.6 & 45.8 & 47.9 \\
570 & 36.3 & 36.9 & 37.3 & 39.4 & 42.7 & 44.8 & 46.7 \\
580 & 35.2 & 35.8 & 36.3 & 37.9 & 40.8 & 42.7 & 44.5 \\
590 & 36.4 & 36.8 & 37.6 & 39.4 & 42.0 & 44.4 & 42.7 \\
\hline
\end{tabular}




$$
k_{\downarrow}(\lambda)=4.61 / z_{Q}(1 \%) .
$$

This depth, also known as the depth of euphotic zone, was proposed by Jerlov [8] in his optical classification of water masses. This value is connected with $z_{90}[4]$ by the following relationship:

$$
z_{90}=\frac{1}{4.61} z_{Q}(1 \%)
$$

Using experimental data taken from Refs. [11-13] we obtained the following connection between euphotic zone depth and effective wavelength:

$$
z_{Q}\{1 \%\}=657.6+1.26 \lambda_{\text {eff }}, \quad\left(464<\lambda_{\text {eff }}<521 \mathrm{~nm}\right),(N=80, r=0.96) .
$$

The data set used to obtain Eq. (11) is shown in Fig. 1.

\section{CONNECTION BETWEEN EFFECTIVE WAVELENGTH OF UPWELLING RADIATION AND INHERENT OPTICAL PROPERTIES OF SEAWATER}

One of the important results of our measurements is a discovery of connection between effective wavelength $\lambda_{\text {eff }}$ and inherent optical properties of the upper layer of the sea. Transfer and distribution of light energy in sea depth is determined by absorption and scattering processes. The accurate description of these processes is very important for overall solution of radiative transfer problems [14-16]. The in-situ experiments often lack complete set of direct measurements of inherent optical properties, especially absorption coefficient $a(\lambda)$ and phase function of

Table 3. Numerical values of coefficients $A_{3}, B_{3}, A_{4}$, and $B_{4}$ in Eqs. (8) and (12).

\begin{tabular}{|c|c|c|c|c|c|c|}
\hline$\lambda, \mathrm{nm}$ & $-A_{3}(\lambda)$ & $B_{3}(\lambda) \cdot 10^{2}$ & $r$ & $-A_{4}(\lambda)$ & $B_{4}(\lambda) \cdot 10^{2}$ & $r$ \\
\hline 410 & 13.24 & 2.52 & 0.97 & 13.91 & 2.64 & 0.97 \\
420 & 13.23 & 2.52 & 0.97 & 13.81 & 2.62 & 0.98 \\
430 & 13.18 & 2.51 & 0.98 & 13.77 & 2.61 & 0.98 \\
440 & 13.06 & 2.48 & 0.98 & 13.48 & 2.55 & 0.98 \\
450 & 12.49 & 2.36 & 0.97 & 12.83 & 2.41 & 0.98 \\
460 & 12.39 & 2.38 & 0.97 & 12.57 & 2.35 & 0.97 \\
470 & 12.19 & 2.29 & 0.97 & 12.45 & 2.32 & 0.97 \\
480 & 11.77 & 2.20 & 0.97 & 11.87 & 2.20 & 0.97 \\
490 & 11.33 & 2.11 & 0.97 & 11.30 & 2.08 & 0.96 \\
500 & 10.43 & 1.93 & 0.96 & 10.41 & 1.90 & 0.96 \\
510 & 8.95 & 1.63 & 0.95 & 8.85 & 1.60 & 0.95 \\
520 & 8.11 & 1.46 & 0.94 & 7.83 & 1.39 & 0.94 \\
530 & 7.70 & 1.38 & 0.93 & 7.42 & 1.31 & 0.93 \\
540 & 7.07 & 1.25 & 0.92 & 6.74 & 1.17 & 0.92 \\
550 & 6.30 & 1.10 & 0.90 & 5.93 & 1.01 & 0.91 \\
560 & 5.60 & 0.96 & 0.88 & 5.20 & 0.86 & 0.89 \\
570 & 5.12 & 0.87 & 0.86 & 4.84 & 0.80 & 0.88 \\
580 & 4.62 & 0.78 & 0.84 & 4.28 & 0.70 & 0.85 \\
590 & - & - & - & 4.30 & 0.72 & 0.86 \\
\hline
\end{tabular}




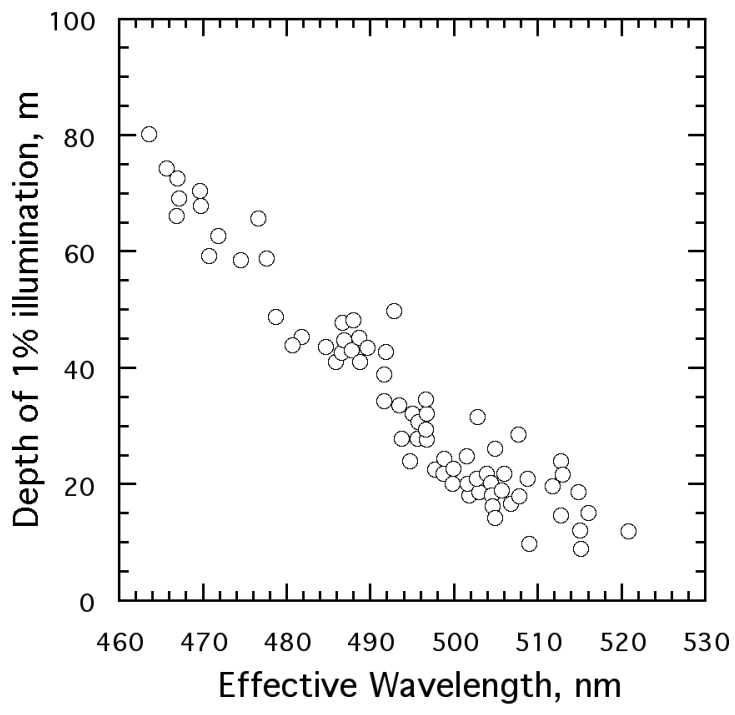

Figure 1. Dependence of euphotic zone depth (depth of $1 \%$ irradiance) on effective wavelength of upwelling radiance from the sea.

scattering $p(\lambda, \vartheta)$, where $\vartheta$ is a scattering angle. This lead to the development of indirect methods to estimate absorption coefficient and phase function of scattering [17]. The method to estimate absorption coefficient is based on a dependence of spectral signatures of upwelling light with spectral dependence of absorption coefficient.

Our analysis of experimental data showed that dependence between absorption coefficient and effective wavelength may be represented as a linear regression in semi-logarithmic scale:

$$
\lg a(\lambda)=A_{4}(\lambda)+B_{4}(\lambda) \lambda_{\text {eff }},
$$

here $a(\lambda)$ (in $\mathrm{m}^{-1}$ ) is an absorption coefficient averaged over subsurface layer. Coefficients $A_{4}$ and $B_{4}$ are given in Tab. 3. Spectral dependence of correlation coefficient for Eq. (12) is similar to the same of Eq. (8) and reaches maximum $r=0.98$ at $\lambda=420 \div 450 \mathrm{~nm}$. Majority of published data on absorption coefficient satisfy Eq. (12).

We should also note that Eqs. (8) and (12) contain values of diffuse attenuation and absorption coefficients averaged over upper sea layer without any precise information on vertical stratification of these quantities.

If we have measurements of vertical profiles of spectral beam attenuation coefficient, we can compute the following spectral depth using following definition:

$$
z^{*}(\lambda)=\int_{0}^{\infty} z \exp \left[-\int_{0}^{z} c\left(\lambda, z^{\prime}\right) d z^{\prime}\right] d z / \int_{0}^{\infty} \exp \left[-\int_{0}^{z} c\left(\lambda, z^{\prime}\right) d z^{\prime}\right] d z
$$

here $c(\lambda, z)$ is a spectral depth profile of attenuation coefficient in $\mathrm{m}^{-1}, z$ is a depth in $\mathrm{m}$. For a homogeneous sea $z^{*}(\lambda)=1 / c(\lambda)$, i. e. $z^{*}$ coincides with spectral optical length of seawater.

The relationship between optical thickness and beam attenuation coefficient allow us to define beam attenuation coefficient averaged over upper layer of the sea using the following definition:

$$
\bar{c}(\lambda)=\int_{0}^{\infty} \exp \left[-\int_{0}^{z} c\left(\lambda, z^{\prime}\right) d z^{\prime}\right] d z / \int_{0}^{\infty} z \exp \left[-\int_{0}^{z} c\left(\lambda, z^{\prime}\right) d z^{\prime}\right] d z .
$$

Experimental measurements show that spectral depth $z^{*}(\lambda)$ has a distinctive spectral shape that makes it 

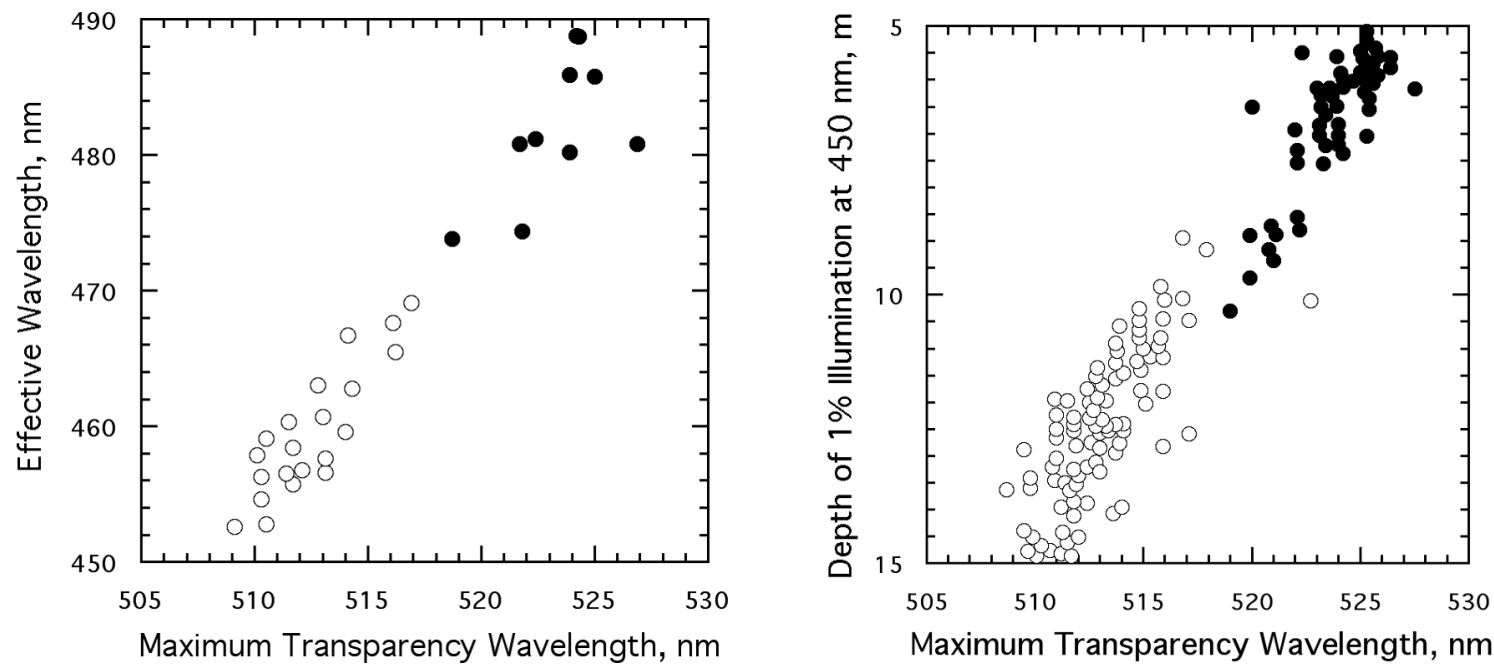

Figure 2 . Dependence of effective wavelength of upwelling sea radiance $\lambda_{\text {eff }}(\Phi)$ (left) and of the depth at which light attenuates e times $z^{*}(450)$ (right) with the wavelength of maximum transmission of water $\lambda_{\text {eff }}\left(z^{*}\right)$.

suitable to be described by the following integral parameter:

$$
\lambda_{\text {eff }}\left(z^{*}\right)=\int_{\lambda_{1}}^{\lambda_{2}} \lambda z^{*}(\lambda) d \lambda / \int_{\lambda_{1}}^{\lambda_{2}} z^{*}(\lambda) d \lambda
$$

Comparison of in situ measurements made in Black and Mediterranean seas showed that $\lambda_{\text {eff }}\left(z^{*}\right)$ correlates both with $z^{*}(\lambda)$ and $\lambda_{\text {eff }}(\Phi)$, where $\Phi$ is any one of $L_{\uparrow}, E_{\uparrow}, R$, or $r$ (See. Fig. 2). We do not give here regressional relationships between above mentioned parameters because a preliminary nature of these investigations.

\section{CONNECTION BETWEEN EFFECTIVE WAVELENGTH OF UPWELLING RADIATION AND CHLOROPHYLL CONCENTRATION IN SEAWATER}

Biological components of natural waters, the phytoplankton and products of its life cycle, play significant role in processes of absorption and scattering of radiation in visible range of spectrum. These processes cause the correlation between chlorophyll content [2] which is a dominant component in seawater and parameters of upwelling radiation. The regressional relationships similar to the following,

$$
C_{c h l}=A_{5} J\left(\lambda_{n}, \lambda_{k}\right)^{B_{5}},
$$

are widely used [18]. here $A_{5}$ and $B_{5}$ depend on the position of wavelengths $\lambda_{n}$ and $\lambda_{k}$ in spectrum. When one or both these wavelength happened to be at the end of decreasing part of the spectrum, it is very difficult to obtain accurate values of color index $J$, and, consequently, accurate values of $C_{c h l}$. This problem was discussed by Hovis et. al. [19]. The authors of Ref. [19] in order to increase accuracy of $C_{c h l}$ determination varied values of $\lambda_{n}$ and $\lambda_{k}$ using different combination of 443, 520, 550, and $670 \mathrm{~nm}$ channels of Coastal Zone Color Scanner. 
The use of effective wavelength $\lambda_{\text {eff }}(\Phi)$ eliminates this shortcoming because this quantity is determined by the most energetic components of the optical spectrum $\Phi(\lambda)$. Our measurements showed that the effective wavelength very well correlates with the concentration of subsurface chlorophyll- $a$. The regression between $C_{c h l}$ (in $\mathrm{mg} / \mathrm{m}^{3}$ ) and effective wavelength (in $\mathrm{nm}$ ) is expressed as follows:

$$
C_{c h l}=\exp \left[0.117\left(\lambda_{\text {eff }}-498.2\right)\right], \quad\left(460<\lambda_{\text {eff }}<520 \mathrm{~nm}\right), \quad(N=74, r=0.91) .
$$

To derive Eq. (17) we used both our data obtained in Black and Mediterranean seas and data obtained from Refs. $[11,20]$.

Determination of chlorophyll concentration is also possible on the basis of connection between $C_{c h l}$ and downward diffuse attenuation coefficient $k_{\downarrow}$. Spectral signature of downward diffuse attenuation coefficient is used by Smith and Backer [10] in their remote sensing model to derive chlorophyll concentration. Determination of $k_{\downarrow}$ using $\lambda_{\text {eff }}$ may be applied to this model and converted to simple engineering equation:

$$
k_{\downarrow}(500)=\exp \left[0.0444\left(\lambda_{\text {eff }}-540.4\right)\right]
$$

Also, by using Eq. (8) with coefficients taken from Tab. 3, and relationships $k_{\downarrow}(500)=1.15 / z_{S}$, and $\lg C_{c h l}=2.37-2.53 \lg \left(z_{S}\right)$ borrowed from Refs. [6, 21], we can obtain the following formula:

$$
C_{c h l}=\exp \left[0.112\left(\lambda_{\text {eff }}-495.3\right)\right]
$$

The coefficients in Eq. (19) are very close to the coefficients of Eq. (17). This circumstance proves validity of dependencies (8) and (17) that connect $\lambda_{\text {eff }}$ with corresponding bio-optical properties.

The level of biological productivity of waters is closely connected with a photosynthesis reaction which consists of absorption of light quantum by a phytoplankton cell to produce organic matter. As a consequence the depth profile of quantum irradiance in productive waters differs significantly from similar profile in oligotrophic waters of open ocean. This difference may be described by Lorenzen formula [22] that connects euphotic zone depth (the depth at which quantum irradiance decreases 100 times) with the subsurface concentration of chlorophyll:

$$
\lg z(1 \%)=3.457-0.297 C_{c h l} .
$$

A combination of Eqs. (11) and (20) allows to obtain values of chlorophyll that are close (but somewhat underestimated) than values produced by Eq. (17). It is necessary to note that Eq. (20) is valid only for very turbid waters with $z_{Q}(1 \%) \leq 30 \mathrm{~m}$ (corresponding values of $\lambda_{\text {eff }} \geq 500 \mathrm{~nm}$ ).

\section{CONCLUSION}

The experimentally derived relationships proposed in this paper proves usefulness of the universal integral parameter of multispectral optical signature of the ocean water, the effective wavelength $\lambda_{\text {eff }}$. By using this

parameter it is possible to significantly diminish flow of primary data that characterized effectively multispectral signatures of the ocean. 


\section{ACKNOWLEDGMENTS}

The authors from the Naval Research Laboratory (NRL) thank continuing support at the NRL through the programs Spectral Signatures 5939-A1 and Hyperspectral Signatures 8028-A1. This article represents NRL contribution $\mathrm{PP} / 7330-01-0067$.

\section{REFERENCES}

1. J. E. Tyler, and R. C. Smith, "Measurements of spectral irradiance underwater," Ocean Sci., 1, (Gordon \& Breach, New York, N.Y., 1970), 103 pp.

2. V. I. Haltrin, "Chlorophyll based model of seawater optical properties," Applied Optics, 38, 6826-6832, (1999).

3. D. B. Judd, and C. Wyszecki, Color in Business, Science and Industry, (John Wiley \& Sons, New York Chichester - Brisbane - Toronto, 1975), 553 pp.

4. V. I. Haltrin, D. R. Johnson, and V. A. Urdenko, "Connection between light field parameters and optical properties of seawater," These Proceedings, Paper No. 4488-A18.

5. N. G. Jerlov, Optical Oceanography, (Elsevier Pub. Co., Amsterdam - London - New York, 1968), 194 pp.

6. V. E. Shemshura, E. I. Afonin, and V. A. Urdenko, Statistical dependencies between certain properties of light fiels in sewater. Part 1: Results of in-situ measurements, 34 pp, (VINIITI Report No. 110-84Dep., Moscow, Russia, 1984), in Russian.

7. N. G. Jerlov, "Optical studies of ocean water," Reports Swedish Deep-Sea Expedition, 3, 1-59 (1951).

8. N. G. Jerlov, "Classification of seawater in terms of quanta irradiance," J. Cons. Int. Explor. Mer., 37 (6), 281-287, (1977).

9. V. N. Pelevin, "Solar radiation in ocean," in Ocean Optics, Vol. 1, A. S. Monin, ed., pp. 268-281, (Nauka, Moscow, Russia, 1983), in Russian.

10. R. C. Smith, and K. S. Baker, "Optical classification of natural waters," Limnol. Oceanogr., 23, 260-267, (1978).

11. A. Morel, et L. Prieur, Analyse spectrale des coefficients d'attenuation diffuse, de reflection diffuse, d'absorption et de retrodiffusion pou diverse regions marines, (Laboratoire d'Oceanographie Physique, Rapp. No. 17, Villfranche-sur-Mer, France, 1975).

12. N. K. Højerslev, Inherent and apparent optical properties of the Northern Sea. Fladen Ground Experiment. - Flex. 75, Rep. No. 32, 39 pp., (Univ. Copenhagen, Inst. Phys. Oceanogr., Denmark, 1977).

13. V. S. Khazanov, and T. Ya. Krayman, "Light-measuring white chloroplast plate," Optico-Mechanical Industry, No. 4, 5-52, (1975), in Russian.

14. V. I. Haltrin, "Self-consistent approach to the solution of the light transfer problem for irradiances in marine waters with arbitrary turbidity, depth and surface illumination," Appl. Optics, 37, 3773-3784 (1998).

15. V. I. Haltrin, "Apparent optical properties of the sea illuminated by Sun and sky: case of the optically deep sea," Appl. Optics, 37, 8336-8340, (1998).

16. V. I. Haltrin, "Diffuse reflection coefficient of a stratified sea," Applied Optics, 38, 932-936 (1999). 
17. V. I. Haltrin,"Empirical algorithms to restore a complete set of inherent optical properties of seawater using any two of these properties," Canadian Journal of Remote Sensing, 26, 440-445, (2000).

18. H. R. Gordon, and A. Morel, Remote assessment of ocean color for interpretation of satellite visible imagery, (Springer Verlag, 1983). (for this model $A_{5}=1.92, B_{5}=1.8, \lambda_{n}=550 \mathrm{~nm}, \lambda_{k}=440 \mathrm{~nm}$ )

19. W. A. Howis, D. K. Clark, F. Anderson, et. al., "Nimbus-7 Coastal Zone Color scanner: System Description and Initial Imagery," Science, 210 (3), 60-63, (1980).

20. J. Joseph, "Untersuchungen über ober- und Unterlichtmessungen im Meere und über ihren Zusammenhang mit Durchsiuchtigkeitsmessungen," Deut. Hydrogr. Zeit., 3, 324-335, (1950).

21. V. E. Shemshura, V. A. Urdenko, and V. I. Fedirko, "On coupling between Secchi disk visibility with concentration of chlorophyll-a in water," Oceanology, 22, 404-407, (1982).

22. C. J. Lorenzen, "Surface chlorophyll as an index of the depth, chlorophyll content and primary productivity of the euphotic layers," Limnol. Oceanogr., 15, 479-480, (1970). 